\title{
Buschke-Löwenstein tumour associated with low-risk human papillomavirus genotypes successfully treated surgically
}

\author{
Dorota Purzycka-Bohdan ${ }^{1}$, Aneta Szczerkowska-Dobosz ${ }^{1}$, Julita Swiatecka-Czaj ${ }^{2}$, Rafal Peksa ${ }^{3}$, Marek Urban $^{1}$, \\ Michal Szczypior ${ }^{4}$, Roman J. Nowicki ${ }^{1}$
}

${ }^{1}$ Department of Dermatology, Venereology and Allergology, Medical University of Gdansk, Gdansk, Poland

${ }^{2}$ Department of Dermatology, Nicolaus Copernicus Hospital, Gdansk, Poland

${ }^{3}$ Department of Pathomorphology, Medical University of Gdansk, Gdansk, Poland

${ }^{4}$ Department of Urology, Medical University of Gdansk, Gdansk, Poland

Giant condyloma acuminatum termed as a Buschke-Löwenstein tumour (BLT) is an invasively slow-growing wart-like skin lesion of the anogenital region [1]. Although the disorder is histologically benign, its management is challenging due to its large size, local tissue destruction, and high recurrence rate [2]. We report a case of genital BLT associated with a lowrisk human papillomavirus (HPV) infection which was treated successfully with radical excision and reconstructive surgery.

A 66-year-old man was admitted to the dermatological outpatient clinic due to a large polypoid lesion in the

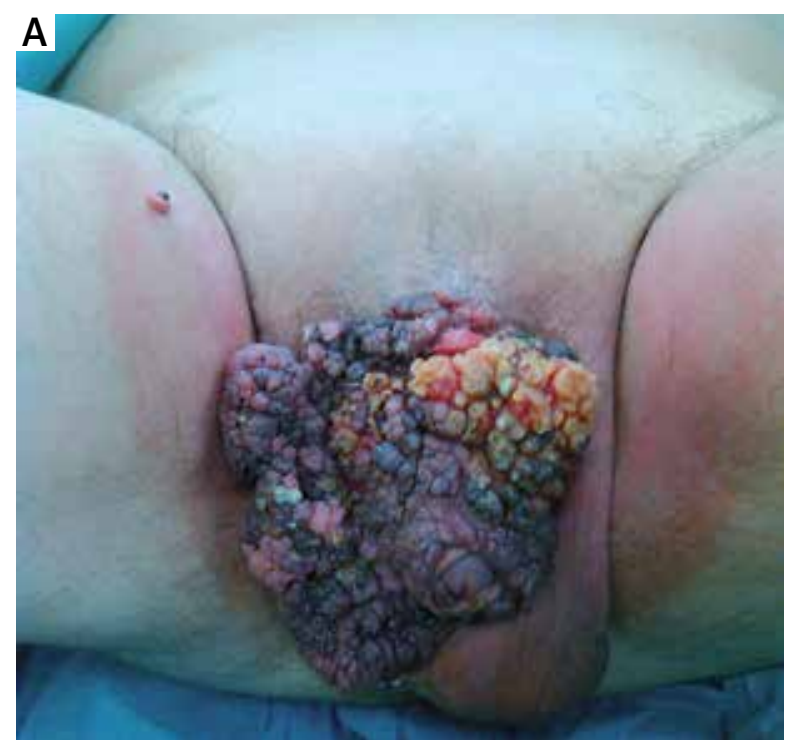

genital area (Figure $1 \mathrm{~A}$ ). The single brownish small wart had first appeared on the penis 19 years before and had grown gradually expanding also the perineal region. The lesion was painless, but caused disfigurement of the penis and made sexual intercourses impossible. Nevertheless, the patient had not sought any therapy. He denied any sexual risk behaviours. The patient was suffering from hypertension, benign prostatic hyperplasia and degenerative joint disease. The family history of skin disorders was negative.

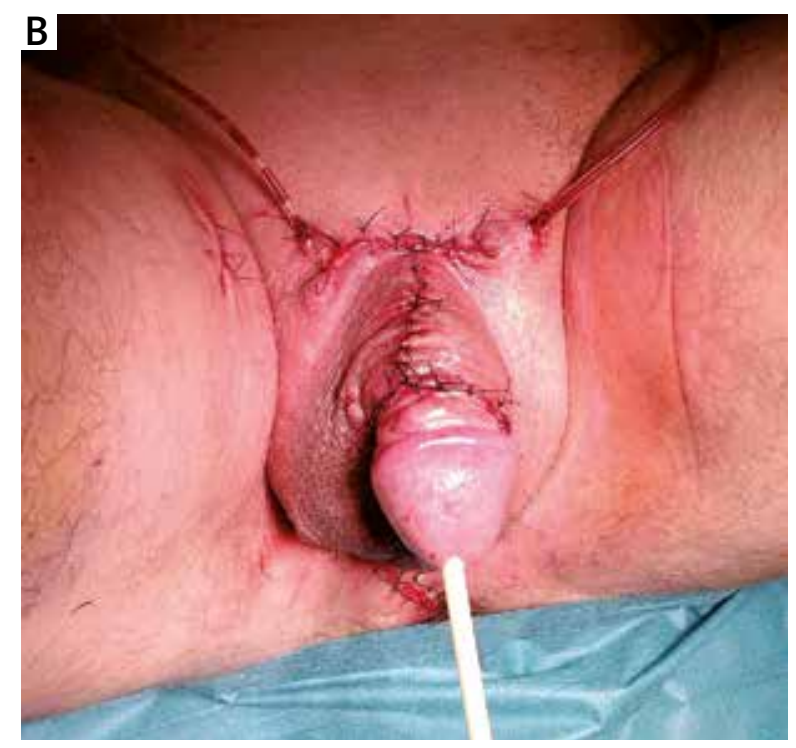

Figure 1. A - Cauliflower-like, large, yellowish-grey papillary tumour covering almost the entire surface of the penis skin and a part of the scrotum; a small "satellite" of the tumour seen on the right thigh; B - the affected region after excisional and reconstructive surgery

Address for correspondence: Dorota Purzycka-Bohdan MD, PhD, Department of Dermatology, Venereology and Allergology, Medical University of Gdansk, 1 a Kliniczna St, 80-402 Gdansk, Poland, phone: +48 5834925 80, fax: +48 583492586 , e-mail: purzycka-bohdan@gumed.edu.pl Received: 27.08.2017, accepted: 11.10.2017. 
In the conducted digital rectal examination, the wall of the anus and anal canal were unaffected. The prostate gland was enlarged, smooth, symmetrical, without palpable changes. Urination was effortless. The mucosa of the glans penis was normal. Lymph nodes were not palpable. The diagnostic tests towards other sexually transmitted diseases was negative. The skin biopsies were taken and the patient was referred to the department of urology for further diagnostics and treatment. Histopathological examination of the genital skin lesion confirmed the clinical suspicion of giant condyloma acuminatum. Moreover, HPV detection by polymerase chain reaction method (PCR) from the biopsy specimens was performed and revealed the presence of HPV 6 and 11 genotypes (Figure $2 \mathrm{~B}$ ).

The therapy consisted of radical excision with the follow-up reconstruction by rotation flap surgery (Figure $1 \mathrm{~B}$ ). The patient was discharged from hospital in good condition with recommendation of antibiotic treatment and daily wound dressings. All the surgical material was forwarded for histopathological examination which confirmed the previous diagnosis of BLT (Figure $2 \mathrm{~A}$ ). No recurrence was observed during 5 months of follow-up.

The giant condyloma acuminatum was originally described by Buschke in 1986 [3] and Löwenstein in 1925 [4] as a penile lesion. The disorder belongs to a group of 'semi-malignant' verrucous carcinomas due to their destructive local evolution [5], however its terminology is still controversial [6]. Some authors consider BLT to be a regional variant of verrucous carcinoma while others classify it as a separate entity [7-9]. Chu et al. analysed 42 known cases of BLT in the English literature and reviewed their behaviour and management [7]. The disease

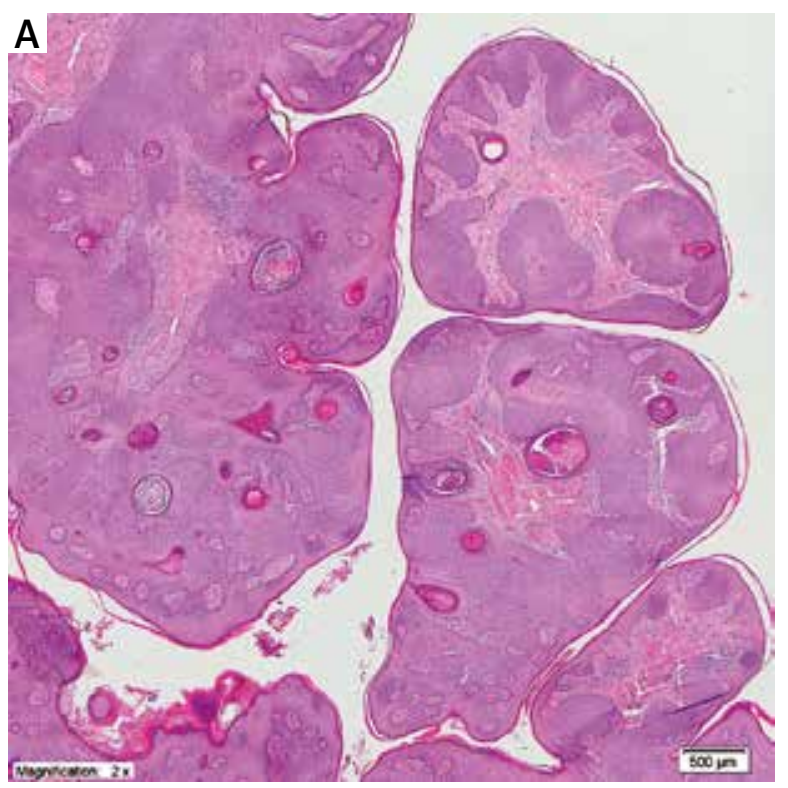

was reported to have a significant rate of recurrence and a risk of malignant transformation to squamous cell carcinoma (SCC) with no distant metastases [7]. Trombetta et al. noticed the presence of neoplastic foci in as many as $50 \%$ of the 52 reports of patients undergoing surgery for BLT [10].

The BLT is considered a relatively rare sexually transmitted disease with an estimated incidence of $0.1 \%$ in the general population [11]. The ratio between women and men is $1: 3$, and the mean age of occurrence is about 50 years [11].

The etiopathogenesis of BLT also remains unclear, however, its association with HPV infection has been highlighted [1]. Low-risk HPV DNA (types 6 and 11) has been most commonly identified in skin samples of BLT, strongly suggesting their pathogenic role in tumour development. The presence of these two HPV types was also detected in our case. The risk factors for BLT also include an HIV infection, homosexuality and immunosuppression [11, 12].

The diagnosis of BLT is usually based on the patient's history, clinical features and histopathological examination. In certain cases, due to the risk of deep local invasion especially in the anorectal region, imaging studies are also required. Before the beginning of therapy, it is important to obtain a skin biopsy. The BLT is a histologically benign disorder, nevertheless careful histopathological examination is necessary to exclude the presence of

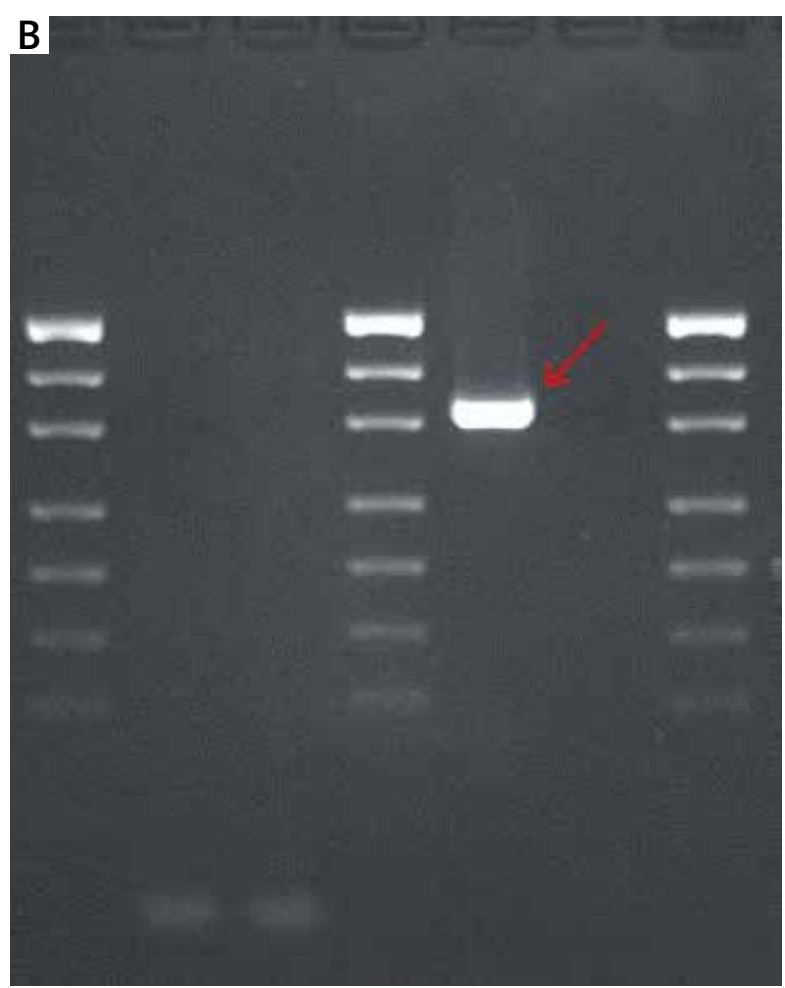

Figure 2. A - The biopsy of the surface of the lesion with arborescent papillae showing prominent fibrovascular cores, surface koilocytosis, and no significant atypia; B - HPV 6 and 11 detection (arrow) with PCR method 
SCC transformation. The histopathological presentation with condyloma features (koilocytosis and fibrovascular cores) but with more prominent bulbous expansion into the underlying tissue and endophytic pattern of growth without presence of atypia is crucial to confirm BLT and exclude other benign or malignant lesions. The BLT of the anogenital region can be misdiagnosed mainly because of the chronic friction and risk of maceration. There are some rarely occurring lesions in the anogenital area clinically similar to BLT such as giant seborrheic keratosis [13].

Early surgical resection of condyloma acuminatum prevents the BLT development [8]. The BLT management is challenging due to its size and degree of local invasion [12]. The tumour should be treated radically as topical chemotherapy, laser resection or cryosurgery are not sufficient in terms of disease control and recurrence. Wide radical excision with the follow-up reconstruction seems to be the treatment of choice, and it was also implemented in our case. Neo-adjuvant chemo-radiation therapy with local excision may be considered in the treatment of BLT with SCC transformation, but also in unresectable or recurrent disease [14]. The effectiveness of adjuvant immunotherapy is still uncertain. Due to a risk of recurrences, follow-up visits are strongly required. In our case, we excluded presence of malignant transformation and so a wide excision and reconstructive surgery exclusively seemed to be the best choice of treatment. Unfortunately, the follow-up period was only 5 months because the patient did not keep his further appointments with the doctor.

The presence of genital BLT certainly causes discomfort, adversely affects sexual life and, therefore, it decreases the quality of patient's life [15]. An early diagnosis, appropriately aggressive therapy and vigilant surveillance are crucial to improve patient outcome. A long-term follow-up period can decrease the risk of recurrence.

In conclusion, we present a case of BLT associated with HPV 6 and 11 genotypes successfully managed surgically. We point out that although several treatment modalities have been proposed, a wide surgical excision with reconstructive surgery seems to be the optimal therapeutic strategy in such disfiguring tumours of the genital region with a risk of relapses and even malignant transformation.

\section{Conflict of interest}

The authors declare no conflict of interest.

\section{References}

1. Braga JC, Nadal SR, Stiepcich M, et al. Buschke-Loewenstein tumor: identification of HPV type 6 and 11. An Bras Dermatol 2012; 87: 131-4.

2. Agarwal S, Nirwal GK, Singh H. Buschke-Lowenstein tumour of glans penis. Int J Surg Case Rep 2014; 5: 215-8.
3. Buschke A. Neisser's Sterokopischer Atlas. Fischer, New York, USA 1986

4. Buschke A, Löwenstein L. Über carcinomähnliche condylomata accuminata des Penis. Klin Wochenschr 1925; 7: 1726-8.

5. Schwartz RA. Verrucous carcinoma of the skin and mucosa. J Am Acad Dermatol 1995; 32: 1-21.

6. Virgilio E, Balducci G, Mercantini P, et al. Perianal giant condyloma acuminatum of Buschke-Loewenstein: a carcinomalike condyloma or a condyloma-like carcinoma? ANZ I Surg 2015; 85: 394-5.

7. Chu QD, Vezeridis MP, Libbey NP, Wanebo HI. Giant condyloma acuminatum (Buschke-Lowenstein tumor) of the anorectal and perianal regions: analysis of 42 cases. Dis Colon Rectum 1994; 37: 950-7.

8. Tas S, Arik MK, Ozkul F, et al. Perianal giant condyloma acuminatum - Buschke-Löwenstein tumor: a case report. Case Rep Surg 2012; 2012: 507374.

9. Ahsaini M, Tahiri Y, Tazi MF, et al. Verrucous carcinoma arising in an extended giant condyloma acuminatum (BuschkeLöwenstein tumor): a case report and review of the literature. J Med Case Rep 2013; 7: 273.

10. Trombetta LJ, Place RJ. Giant condyloma acuminatum of the anorectum: trends in epidemiology and management: report of a case and review of the literature. Dis Colon Rectum 2001; 44: 1878-86.

11. Atkinson AL, Pursell N, Sisay A. The giant condyloma (Buschke-Löwenstein tumor) in the immunocompromised patient. Case Rep Obstet Gynecol 2014; 2014: 793534.

12. Gole GN, Shekhar T, Gole SG, Prabhala S. Successful treatment of Buschke-Löwenstein tumour by surgical excision alone. J Cutan Aesthet Surg 2010; 3: 174-6.

13. Sudhakar N, Venkatesan S, Mohanasundari PS, et al. Seborrheic keratosis over genitalia masquerading as BuschkeLowenstein tumor. Indian I Sex Transm Dis 2015; 36: 77-9.

14. Indinnimeo M, Impagnatiello A, D’Ettorre G, et al. BuschkeLöwenstein tumor with squamous cell carcinoma treated with chemo-radiation therapy and local surgical excision: report of three cases. World J Surg Oncol 2013; 11: 231.

15. Ciobanu AM, Popa C, Marcu M, et al. Psychotic depression due to giant condyloma Buschke-Löwenstein tumors. Rom J Morphol Embryol 2014; 55: 189-95. 